\title{
Recomposition des systèmes agricoles de moyenne montagne. Mutations et perspectives. Le cas de l'Aveyron
}

\section{Betty Wampfler}

\section{Résumé}

Le débat sur le rôle de l'agriculture qui oppose une agriculture compétitive à une "agriculture différente", réactualisé par la réforme de la PAC, se pose avec une acuité renforcée dans les zones défavorisées. A partir de l'exemple de l'agriculture aveyronnaise, cette étude s'interroge sur les transformations en cours au niveau des systèmes productifs de ces zones, sous l'impact de la "crise" et des ajustements de la politique agricole. L'hypothèse de l'émergence d'un nouveau référentiel agricole comme solution de sortie de crise est ensuite étudiée.

\section{Abstract}

The debate about the functions of agriculture wich set an competitiv agriculture against a "different agriculture", is re-actualized by the reform of European Politic of Agriculture. This question is still more important in less favourized areas. This study in focused of the transformation of farming systems of such areas face of the agriculture crisis and the reform of European Politic of Agriculture.

\section{Citer ce document / Cite this document :}

Wampfler Betty. Recomposition des systèmes agricoles de moyenne montagne. Mutations et perspectives. Le cas de l'Aveyron. In: Économie rurale. N²24, 1994. pp. 52-57;

doi : https://doi.org/10.3406/ecoru.1994.4693

https://www.persee.fr/doc/ecoru_0013-0559_1994_num_224_1_4693

Fichier pdf généré le 08/05/2018 


\section{Q 2 ecomposition des systèmes Mutations et perspectives. Le cas de l'Aveyron}

$\mathbf{L}$ es années 80 ont été, pour l'agriculture, une période de "crise", de ruptures, d'autant plus profondes que les décennies précédentes avaient été marquées par l'euphorie de la modernisation. En amont des problèmes conjoncturels, c'est, plus fondamentalement, le "modèle de développement" qui est remis en cause, la fin des "modèles" qui est annoncée, et avec elle, le nécessaire passage d'un système perçu comme cohérent, à un "autre chose", dont les contours sont mal définis et ne font, pour l'instant, l'objet d'aucun consensus (Pour, 1991). Ranimé par la réforme de la PAC, le débat ne date pas d'aujourd'hui. Le terme même de "modèle", renvoie à sa structuration, autour de -schématiquement- deux conceptions de l'agriculture.

Pour les théoriciens du "productivisme" (Bergmann, 1989), l'agriculture est un secteur économique en voie de normalisation au sein de la formation économique globale. La compétitivité doit être son objectif prioritaire, dans l'optique d'une insertion renforcée dans l'internationalisation des échanges. Pour ce faire, le "productivisme", l'accroissement de la productivité obtenu par l'intensification des facteurs rares travail et terre, la spécialisation et la concentration des structures, sont les seules voies possibles (il n'y a donc pas lieu de parler de "modèle"). La baisse induite du nombre d'exploitations doit être encouragée et non freinée, car elle s'inscrit dans la logique de l'affectation optimale des ressources entre les différents secteurs productifs.

A cette conception strictement économique de l'agriculture, s'opposent des questionnements sur des "agricultures différentes", aux contours plus difficiles à cerner parce que multiples, mais se rejoignant dans la contestation du "modèle dominant" de la modernisation agricole française et l'analyse de voies alternatives. La remise en cause du "modèle dominant" s'appuie sur des arguments économiques (baisse de productivité des intrants, de la valeur ajoutée agricole et des revenus des agriculteurs), sociaux (persistances des disparités au sein du secteur) et sociétaux (préoccupation sur la qualité de l'alimentation et du cadre de vie) (Bonny, 1980). Les voies alternatives envisagées présentent des degrés variables de rupture avec le "modèle dominant", chacune d'elles se situe en contre-point d'une caractéristique contestée du "productivisme", mais l'ensemble, censé s'articuler à une nouvelle demande sociale, ne définit pas -pas encore ? - un nouveau référentiel de développement, ou un projet cohérent et socialement soutenu sur lequel des acteurs ou des organisations pourraient se positionner.
Ce débat s'impose avec une acuité renforcée dans les zones défavorisées. Condamnée à un déclin rapide et sans appel par les thèses productivistes (Bergmann, 1989), l'agriculture de ces zones reste, en tant que segment du monde rural, un objet de préoccupation et de recherche pour les tenants d'un développement territorial plus équilibré.

C'est dans le contexte de ce débat que nous nous proposons d'étudier les transformations en cours dans l'agriculture de zones défavorisées, d'analyser les formes de passage entre les anciennes et les nouvelles conditions de l'activité agricole, en liaison avec l'évolution de la PAC et du contexte socio-économique englobant.

\section{Questionnement de recherche et grille d'analyse}

L'analyse porte sur les choix économiques des ménages agricoles, insérés dans un système productif localisé (Benko, Lipietz, 1992) et confrontés à un cadre de contraintes changeant. Les périodes de rupture de cohérence, et de recomposition, mettent en jeu, au niveau des choix économiques des acteurs, la référence au marché, mais aussi les routines, les capacités d'apprentissage, la diversité des modes d'insertion économique et institutionnelle... La volonté de prendre en compte ces différents éléments du cadre de contraintes dans lequel s'effectuent les choix des ménages agricoles, nous conduit à proposer une grille d'analyse combinant des éléments théoriques empruntés à l'analyse systémique, à l'économie rurale, à l'économie des institutions, et à l'analyse des politiques publiques.

Les ménages agricoles sont appréhendés dans leur double appartenance, sectorielle et territoriale, chacune de ces sphères se décomposant en "mésosystèmes" représentant autant de niveaux d'insertion potentielle des ménages.

La sphère sectorielle, régulée par l'institution politique agricole, est fortement structurée par les filières de production, mésosystèmes (De Bandt, 1988; Valceschini, 1991), dans lesquels les entreprises et organismes économiques coopératifs sont des éléments d'intermédiation entre les exploitations agricoles et le marché. Les organisations professionnelles agricoles sont appréhendées dans leur double rôle de médiateurs entre les exploitations, la politique agricole et la formation socio-économique globale, et d'institution régulant le système productif agricole, notamment par la production de "modèles", de normes. 
Les frontières de la sphère d'insertion territoriale des ménages agricoles sont plus diffuses et renvoient à la difficulté de cerner le "local" (Bodiguel, 1986) : communes, départements, régions, mais aussi "bassins de vie", "bassins d'emploi"... dont la régulation fait l'objet de différents niveaux de politiques territoriales.

Enfin, à un niveau qui peut n'être ni sectoriel, ni territorial, les systèmes de réscaux (ćconomiques, sociaux) représentent une forme d'insertion potentielle des ménages agricoles qui peut être appelée à se développer (Pecqueur, 1987).

Les arbitrages économiques des ménages agricoles sont alors considérés comme la résultante d'une logique micro-économique d'optimisation du revenu avec minimisation des risques (Boussard, 1992), modulée par le mode et le degré d'insertion dans les sphères sectorielle et territoriale, les opportunités qu'elles offrent, les règles et normes qu'elles génèrent (Touzard, 1993).

Ces formes d'insertion sont historiquement évolutives : après la seconde guerre mondiale, le processus de modernisation de l'agriculture a contribué à renforcer l'insertion sectorielle et à laminer l'insertion territoriale antérieure des exploitations agricoles. Cette structuration sectorielle s'est opérée sur la base d'une combinaison de principes micro-économiques (augmentation de la production, intensification des facteurs travail et terre par incorporation croissante de capital, spécialisation, concentration des structures) et d'une volonté corporative d'organisation "verticale", tant sur le plan économique que syndical. Les lois de modernisation agricole (1960-19621966) et la Politique Agricole Commune, PAC, en constituent la base institutionnelle et le "productivisme", institué en modèle normatif au sein de la "profession" agricole (Coulomb, Nallet, 1981), le référentiel.

Par "référentiel", nous entendons donc, la combinaison d'une forme de production, d'organisation, et d'une "norme" instituée, qui confere sa cohérence à l'ensemble (Muller, 1990). Si la réussite de la modernisation de l'agriculture a été liée pendant trois décennies à la convergence du référentiel sectoriel adopté avec les besoins et ressources de la formation économique globale, la crise agricole peut être interprétée comme une rupture de cohérence entre ces deux référentiels (Muller, 1989, 1990). Les ajustements de la PAC, puis sa réforme en 1992, constituent des voies de régulation institutionnelles visant à remettre en cohérence le référentiel sectoriel et le référentiel global.

Notre questionnement de recherche porte sur les formes de cette remise en cohérence, dans le cadre spécifique des zones défavorisées.

Peut-on percevoir, à travers les stratégies d'adaptation mises en œuvre, au-delà d'expériences ponctuelles "d'agriculture différente", l'émergence d'un référentiel alternatif, susceptible, à travers de nouveaux modes d'insertion socio-économiques, de réorganiser durablement les systèmes agricoles des zones défavorisées, et de limiter leur déclin annoncé ?

L'articulation entre référentiels sectoriels et référentiel global étant l'objet des politiques publiques (Muller, 1990), quelles sont, à cet égard, les ressources offertes par la nouvelle PAC, et les politiques rurales en gestation?

\section{Éléments de résultats}

\section{Les tendances lourdes de l'évolution agricole pendant la période d'ajustement de la PAC (1980-1990)}

Elles ont été abordées par une enquête portant sur les 2500 exploitations du Ségala, petite région emblématique de la modernisation agricole aveyronnaise. Effectuée une première fois en 1986, et réactualisée en 1992, selon la même méthodologie, cette enquête a permis de constituer une base de données diachronique, interprétée à l'aide de techniques statistiques multidimentionnelles.

\section{Les choix effectués par les ménages agricoles}

Trois types de stratégies ont été observés :

- Les stratégies de retrait du secteur (partiel ou total) sont essentiellement le fait d'agriculteurs âgés, sans succession. Elles prennent des formes traditionnelles (disparition d'exploitation (12\% des exploitations entre 1986 et 1992), décapitalisation lente par abandon d'ateliers ou de volumes (28\% des exploitations), vente d'herbe $(11 \%)$..., mais ont été aussi des réponses à des incitations institutionnelles (préretraite, cessations laitières). Des formes de retrait partiel (double-activité du chef d'exploitation, mais surtout activité extérieure de l'épouse) ont progressé dans des catégories d'exploitations encore actives.

- Les stratégies de diversification (20\% des exploitations) se sont développées dans des voies proches du référentiel initial (essentiellement dé-spécialisation des exploitations bovin-lait, par mise en place d'un second atelier, troupeau viande ( $55 \%$ des ateliers crées entre 1986 et 1992), hors sol (20\% des créations), cultures plus spécialisées, semences, tabac, $(20 \%)$. Les voies plus innovantes sont restées marginales en terme de nombre et de poids économique dans le secteur (5\% seulement parmi les 2500 exploitations enquêtées ont une activité de tourisme ou de transformation/vente directe, en 1992).

- Les stratégies dominantes sont celles de la résistance dans le référentiel productiviste. Le maintien ou l'augmentation du revenu ont été recherchés

- par l'augmentation du volume de production (+ $12 \%$ entre 1986 et 1992, à l'échelle de notre échantillon) ;

- par l'intensification des facteurs travail et terre (augmentation des rendements en lait par vache, augmentation des surfaces irriguées, des surfaces en maïs ensilage ; production de lait de brebis en hors sol, intensification de la production porcine...); - par la concentration des structures et des volumes. Dans notre échantillon, $48 \%$ des exploitations augmentent leur volume de production entre 1986 et 1992 , de $+58 \%$ par rapport au niveau 1986. De même, $31 \%$ des exploitations se répartissent le foncier libéré pendant cette période.

Cette dernière stratégie s'est avérée dominante car les ajustements de la PAC n'ont pas fondamentalement remis en cause l'intérêt d'un développement "productiviste", même si ses résultats économiques se dégradent : l'augmentation de volume, l'intensification du travail et du sol, la concentration des moyens de production restent des voies rentables pour maintenir ou améliorer le revenu, dans un contexte de petites structures. 
La réforme de la PAC, intervenue au terme de cette période, est-elle porteuse d'une alternative susceptible de renouveler les formes de réponse des acteurs face à la "crise" agricole?

\section{La réforme de la PAC est-elle de nature à modifier ces tendances lourdes ?}

Les analyses prospectives sur l'impact de la réforme de la PAC ont été menées, soit à des niveaux macro-économiques (Mahé, Guyomard, 1991 ; Loyat, 1991), soit au niveau des systèmes de production (INRA, 1992). Nous nous proposons d'apporter un éclairage complémentaire à ces travaux par une analyse prenant en compte la diversité des exploitations au niveau d'un territoire, leur mode d'insertion socio-économique et leurs stratégies d'adaptation à la "crise", antérieures à la réforme.

Dans quelle mesure la réforme de la PAC ébranle-t-elle le référentiel sectoriel?

En quoi change-t-elle le cadre des contraintes dans lequel s'effectuent les choix des ménages agricoles?

\section{Impact de la réforme sur les recettes et les revenus agricoles des exploitations}

L'impact de la réforme a été simulé à partir d'une typologie d'exploitations aveyronnaises, sur la base des mesures 1996. Les recettes des systèmes bovin-lait ( $22 \%$ des exploitations aveyronnaises) sont peu modifiées par ces mesures (de $5000 \mathrm{~F}$ à $+20000 \mathrm{~F}$, en fonction de la part de maïs et céréales dans l'assolement). Les systèmes bovin viande sont très diversement touchés, les plus pénalisés étant les plus intensifs (chargement $>2 \mathrm{UGB} / \mathrm{ha}$ ) et ceux ayant une part de cultures de vente importante. Les plus favorisés ( $<1 \mathrm{UGB} / \mathrm{ha})$ bénéficiant de la prime à l'herbe, voient leurs recettes s'améliorer substantiellement (jusqu'à $+50000 \mathrm{~F}$ ). Ils sont peu nombreux dans la région Ségala ( $8 \%$ des exploitations), mais plus fréquents dans les zones du Nord et de l'Est aveyronnais. Les systèmes mixtes lait viande ne sont pas pénalisés, quand ils peuvent bénéficier de la prime à la vache allaitante (quota $<120000$ l). Les systèmes ovins sont d'autant plus gagnants que la part de céréales autoconsommée est importante et que le chargement est inférieur à 1. Mais la présence fréquente de cultures de vente dans ces systèmes les place aussi parmi les plus pénalisés (jusqu'à - $40000 \mathrm{~F}$ ). Les systèmes de grandes cultures sont par contre fortement pénalisés (de -40 à $-60000 \mathrm{~F}$ ), mais sont peu fréquents en Aveyron.

Globalement, la réforme pénalise peu les systèmes aveyronnais ; si elle améliore les recettes des exploitations les plus extensives, elle ne permet pas de compenser les pertes de revenus intervenues pour la viande notamment, en 1990.

Cependant, la question de la pérennité des équilibres observés est posée : d'une part parce que la compensation par les primes ne joue pas, en moyenne, au-delà de $15 \%$ de baisse des prix ; d'autre part, parce que la structure des revenus évolue vers une plus forte dépendance par rapport à l'aide publique. La part des primes dans le revenu disponible final varie de manière forte selon les catégories de producteurs et peut atteindre $100 \%$ du revenu disponible final, dans les petites exploitations spécialisées en viande bovine ou ovine. la question de la pérennité de l'aide est donc cruciale pour plus de $30 \%$ des exploitations de notre échantillon..
Impact sur les pratiques d'intensification/extensification

La réforme crée des opportunités pour l'extensification. Elle consolide les exploitations déjà extensives, dont certaines étaient antérieurement à la limite de la viabilité (tableau 1). Elle peut favoriser le passage de "l'intensif" à l'extensif pour des exploitations proches du seuil discriminatoire de 1,4 UGB/ha. On observe des acquisitions foncières de plus en plus nombreuses répondant à cette stratégie. Par rapport aux justifications données à l'extensification (maîtrise de la production, protection de l'environnement), l'intérêt de ces stratégies pose question, dans la mesure où elles sont souvent combinées à des pratiques productives très contrastées, les bonnes terres de l'exploitation restant fortement intensifiées, les moins fertiles étant dévolues à l'extensif. Les nouveaux règlements limitent aussi dans certains cas, l'intérêt économique des productions en hors sol. Par contre, l'intérêt de l'intensification, au niveau où elle est pratiquée localement, est préservé pour les systèmes laitiers intensifs : les surfaces en maïs ensilage donnent lieu à prime, les surfaces irriguées bénéficient d'une compensation renforcée...

Tableau 1. Incidence de la réforme de la PAC sur une exploitation déjà "extensive" avant 1992

\begin{tabular}{lrr}
\hline & $\begin{array}{r}\text { Situation } \\
1992\end{array}$ & $\begin{array}{r}\text { Situation } \\
\text { après } \\
\text { réforme PAC }\end{array}$ \\
\hline Production & $171148 \mathrm{~F}$ & $146636 \mathrm{~F}$ \\
Primes & $55700 \mathrm{~F}$ & $123356 \mathrm{~F}$ \\
Recettes & $226848 \mathrm{~F}$ & $266992 \mathrm{~F}$ \\
Charges & $146129 \mathrm{~F}$ & $146129 \mathrm{~F}$ \\
Excédent brut d'exploitation & $80719 \mathrm{~F}$ & $120863 \mathrm{~F}$ \\
Revenu disponible & $37817 \mathrm{~F}$ & $77961 \mathrm{~F}$ \\
Part des primes ds le disponible & $147 \%$ & $158 \%$ \\
\hline
\end{tabular}

\section{Impact de la réforme sur la concentration des moyens de production}

Droits à prime, droits à produire, quels enjeux ?

Le facteur le plus déterminant en matière de restructuration est probablement la création d'un droit à prime individuel par exploitation, fixé sur la base du cheptel antérieurement détenu. Les simulations micro-économiques montrent que, au-delà de la différence juridique immédiate, le droit à prime est quasi équivalent à un droit de production : en système viande, par exemple, la marge brute par hectare de surface fourragère, hors prime, couvre tout juste le fermage et les charges de structures liés à ces hectares.

Au niveau des acteurs agricoles, l'apprentissage des quotas lait de vache aidant, le droit à prime a été immédiatement assimilé à un droit à produire : le marché des vaches allaitantes s'est emballé entre juin et décembre 1992, le mot d'ordre des organisations professionnelles a été de constituer les références départementales les plus larges possible... Derrière ces stratégies, deux enjeux sont perceptibles : maximiser l'aide publique perçue, mais aussi préserver des marges de développement potentiel des volumes, au niveau des exploitations et du système productif départemental. Dans ce contexte, le transfert des droits à produire devient un cnjcu déterminant dans la régulation sectorielle. Dans le cas des quotas laitiers, le droit à produire est rattaché au foncier. $\mathrm{Si}$ cette mesure permet de limiter les délocalisations de produc- 
tion, elle a au niveau du système productif départemental, trois types de conséquences:

- Elle confère un intérêt renouvelé à la concentration foncière, puisqu'elle met en jeu, et le foncier, et les droits à produire.

- Elle provoque un renchérissement du foncier dont le prix inclut de fait, les droits à produire associés à la terre.

- Elle induit une dualisation du territoire, entre les terres liées à des droits à produire et celles qui en sont dépourvues.

\section{Vers des "clubs de production" ?}

Notre hypothèse est que la réforme confère une nouvelle rigidité au secteur agricole, en renchérissant l'accès à la plupart des grandes productions d'élevage et en attribuant à chaque exploitation une "licence" de production (qui devient une rente, quand le détenteur donne des terres en fermage, à un prix revalorisé par les droits à produire qui y sont attachés). Elle peut ainsi induire la création de "clubs de production" fermés, dans lesquels la compétition pour l'acquisition des droits à produire va se renforcer et accélérer la concentration des structures.

Au total, si la réforme, appliquée aux zones défavorisées, ouvre bien quelques portes, vers l'extensification notamment, elle ne remet pas fondamentalement en cause le reférentiel "productiviste". Elle consolide un développement fondé sur la concentration des structures et peut être considérée, à ce titre, comme un outil adéquat pour la sélection des exploitations "les plus performantes" de demain.

\section{Vers un nouveau référentiel ?}

Le problème de l'évolution de l'agriculture des zones défavorisées reste donc entier. Ni le référentiel sectoriel, ni la réforme de la PAC, n'offrent un cadre de réponse permettant de contenir le recul de ces zones. Par contre, toutes les stratégies d'innovation observées sur des périodes récentes, se réferent à cet objectif.

Peut-on percevoir dans ces stratégies d'innovation récentes des ménages agricoles et les dynamiques qu'elles suscitent, l'émergence d'un "référentiel alternatif", susceptible, à travers de nouveaux modes d'insertion socio-économique, de favoriser une recomposition durable des systèmes agricoles des zones défavorisées et de limiter ainsi leur déclin annoncé ?

\section{Dans les années récentes, un changement d'ambiance...}

Jusqu'à la fin des années 80 , les stratégies d'innovation sont limitées, isolées et laissées à l'initiative individuelle, dans le système productif agricole observé (voir le chapitre "Les tendances lourdes"). A partir des années 1989, 1990, alors que la "crise" agricole se renforce (baisse des prix de la viande, menace imprécise de réforme de la PAC...), l'ambiance change, les expériences innovantes se multiplient : recherche d'autonomie et d'économie, nouvelles combinaisons productives (associations d'exploitations ou d'ateliers de production, groupement d'employeurs...) ; formes d'organisation innovantes (CUMA employant un salarié artisan, filières courtes entre réseaux de producteurs et d'artisans locaux) ; productions nouvelles ou redécouvertes (fruits, tabac, semences...) ; démarches de qualité engagées en filière; démarches de "qualité fermière" (production fermière, transformation, vente directe), tourisme vert, services...

Dans la plupart des cas, ces innovations interviennent en complément d'une production traditionnelle, en réponse à un problème de revenu insuffisant. Elles correspondent à un "aménagement" du référentiel antérieur, bien plus qu'à sa remise en cause globale. Les cas d'entreprises rurales, développées d'emblée et exclusivement autour d'une production ou activité innovantes, restent limités. Les résultats économiques dégagés sont variables, allant de simples gains "de sécurité" dans des systèmes antérieurement exposés à des pertes importantes par une forte dépendance extérieure, à des gains effectifs de revenus de l'ordre de 30 à $40 \%$, dans des cas de création d'activités. Dans tous les cas étudiés, l'innovation induit une réorganisation du système d'exploitation, et les résultats dégagés sont notamment fonction de la qualité du compromis intervenu entre les activités antérieures et les innovations (en terme de travail, d'investissements...)

\section{Vers un nouveau référentiel ?}

Ces innovations représentent toutes, à des degrés variables, une rupture avec un ou plusieurs des éléments du référentiel sectoriel ("dés-intensification", "dé-spécialisation", rupture avec l'exploitation familiale, avec le cloisonnement sectoriel, ouverture sur de nouvelles fonctions...). Traduisent-elles pour autant, l'émergence d'un nouveau référentiel, de nouvelles cohérences susceptibles d'assurer un développement différent de l'agriculture? Rappelons que la notion de référentiel utilisée, se réfère à la combinaison entre des formes de production (ou d'activités), des formes d'organisation et d'un "paradigme", d'une "norme" donnant sa cohérence à l'ensemble. En quoi cette définition est-elle applicable aux innovations observées?

L'augmentation de la productivité du travail reste un facteur déterminant dans les innovations, mais elle est recherchée par d'autres voies, notamment l'augmentation de la valeur ajoutée. De plus, d'autres objectifs peuvent intervenir dans ces choix : diminuer les risques dans les systèmes très dépendants d'achats extérieurs, mieux "maîtriser son produit", dégager du temps libre, assurer une meilleure qualité de vie, s'ouvrir à un monde non agricole... A la spécialisation, à l'intensification par l'incorporation croissante d'intrants et de capital, au principe de maximisation régissant les stratégies productives antérieures, les expériences innovantes opposent la combinaison de différents éléments (productions, activités, stratégies) et le principe d'optimisation du système d'exploitation, fondé sur le compromis et une valorisation renforcée des ressources locales. Alors que les choix économiques antérieurs s'inscrivaient en marge du marché, dans un cadre de garantie de débouchés illimités à prix soutenus, nombre d'innovations reposent sur une forte insertion dans le marché et des formes de flexibilité peu développées jusque-là dans le secteur agricole (en termes de choix de productions et de techniques, d'organisation du travail, d'investissements...). Enfin, nombre de ces stratégies innovantes sont caractérisées par l'ouverture sur de nouvelles formes d'insertion, territoire ou réseaux. Deux types d'opportunités y sont exploitées : les ressources locales en terme de produits, savoir-faire, traditions... ; les synergies crées par la combinaison de différentes actions innovantes, à l'échelle d'un territoire (par exemple, l'installation d'une école de danse accueillant des stagiaires sur le week-end permet à une ferme-auberge voisine un fonctionnement régulier et à des producteurs fermiers locaux d'asseoir un réseau de vente par correspondance...). 
A l'instar de ce que $\mathrm{N}$. Eizner relevait déjà dans le discours des Etats généraux du développement agricole (Eizner, 1985), ce "retour au local" ne traduit pas ici, un repli sur des valeurs passéistes, mais plutôt la volonté d'inscrire des choix économiques dans une dimension qui semble plus maitrisable par l'individu (au contraire, par exemple, des filières agro-alimentaires, même coopératives, qui sont perçues par nombre d'innovateurs comme des structures laissant d'autant moins de pouvoir au producteur que leurs directions s'éloignent géographiquement).

Globalement, c'est sur une nouvelle complexité et sur un renouvellement de la conception du "métier" d'agriculteur que débouchent ces éléménts de recomposition. Ce nouveau référentiel offre-t-il des opportunités à une fraction plus large de ménages agricoles que le référentiel sectoriel antérieur?

Certes, il permet de consolider des exploitations qui pour des raisons conjoncturelles ou structurelles louvoient autour du seuil de viablité dans le référentiel productiviste. Il permet des installations sur des structures qui ne seraient pas viables, au sein de la seule économie de production. Mais il reste malgré tout très sélectif. D'une part en termes de capacité d'investissement, d'autre part en terme de compétences. Les résultats de l'analyse des démarches qualité, par exemple, incitent à la prudence. Dans la qualité-filière, les niveaux de technicité requis sont tels que seul un nombre limité d'exploitations, déjà "performantes" dans le système antérieur, y ont actuellement accès et en retirent durablement des bénéfices. Dans la qualité-fermière, le tourisme vert et les services, c'est la nature des compétences requises qui est sélective... D'où l'importance, d'une part, des procédures d'appui au financement et d'autre part, des processus d'apprentissage, que l'encadrement institutionnel peut favoriser.

\section{Quelles insertions institutionnelles pour ces innovations?}

- Les innovateurs sont-ils organisés? Si l'émergence d'un référentiel alternatif semble acquise, sa transcription institutionnelle reste embryonnaire : des organisations existent, pour certaines depuis une dizaine d'années (Fermiers du BasRouergue, Coopérative Jeune Montagne...), mais pour la plupart plus récentes ( $\mathrm{GIE}$, groupements de producteurs...). Leur finalité est l'acte commercial, bien plus que la défense ou promotion de voies de développement innovantes. Quand celles-ci sont assurées, souvent d'ailleurs par les mêmes acteurs, c'est au sein des organisations syndicales minoritaires (Confédération paysanne essentiellement), ce qui évidemment n'a pas favorisé la prise en compte de l'innovation par les organisations sectorielles majoritaires.

- Au niveau sectoriel, cependant, le renforcement de la "crise", aux niveaux macro et micro-économiques, ainsi que la multiplication des expériences d'innovation échappant à l'emprise "professionnelle", ont fini par déstabiliser le discours monolithique des organisations agricoles majoritaires locales. L'intégration des innovations y est cependant d'autant plus difficile que celles-ci sont éloignées du référentiel sectoriel antérieur (Projet AGIR, 1990-1993) : "mieux gérer" est devenu un cheval de bataille professionnel local ; la qualité négocićc dans lc cadre d'une inter-profession appelle à des compromis déjà plus difficiles à concéder ; la qualité fermière et la vente directe échappent délibérément à une régulation centralisée et verticale ; quant aux innovations portant sur l'environnement -qui, pour l'instant, apparaissent peu dans les stratégies des ménages- elles font l'objet de démarches résolument corporatistes, excluant d'emblée toute ouverture réelle à d'autres partenaires.

A l'heure actuelle, tout en restant centrées sur le tryptique "grandes productions d'élevage - entrepreneur agricole performant -filières verticales puissantes" (PSAD, 1993), les organisations agricoles majoritaires se sont ouvertes aux activités innovantes, en constatant, d'une part que nombre d'entre elles pouvaient coexister avec, voire consolider, le référentiel sectoriel antérieur ; et que, d'autre part, s'ouvrir en ce sens, était encore le moyen le moins dommageable pour s'épargner un débat autrement plus déstabilisant sur la représentation institutionnelle, la gestion des droits de production, etc.

Cependant, toute action efficace en la matière suppose l'abandon de quelques certitudes bien ancrées : spécificité et unité agricole, primauté de la gestion centralisée verticale, hégémonie de l'agricole sur le rural... Autant de "révolutions culturelles" dont la maturation est difficile, lente et aléatoire, et laisse d'autant plus de place, auprès des innovateurs, pour de nouveaux partenaires et de nouveaux outils institutionnels.

Nouveaux partenaires, nouveaux outils : les exemples analysés montrent une grande variété de partenaires (institutions nationales et régionales (INAO, organismes de certification, par ex.), collectivités territoriales et locales, associations de ruraux, groupes écologistes locaux...) et d'actions (bergerierelais, création de marchés de pays, de micro-filières, de "produits touristiques ruraux", certification d'exploitation...).

Ces nouveaux partenaires sont insérés à différents niveaux de la sphère territoriale, mais aussi au sein de réseaux qui, tout en ayant un ancrage territorial, se veulent éléments de médiation entre le local et la société englobante (associations comme Relier, Civam...). L'agriculture est ici appréhendée comme un élément du "système rural". La mise en cohérence de sa dynamique avec celles des autres éléments de ce système secteurs secondaire et tertiaire, démographie, paysage..., fait l'objet des "politiques locales".

Les frontières de ces "systèmes ruraux" évoluent d'une expérience observée à l'autre (commune, district, canton, bassin de vie...). De même, le degré d'élaboration et le contenu des "politiques locales" varient. Les actions réalisées sont souvent disparates, peu coordonnées, liées à la dynamique entrepreneuriale d'un individu plus qu'à une expression collective de changement.

Peut-on lire dans ces "politiques locales" un cadre "porteur de sens", dans lesquels des projets de développement pourraient durablement s'insérer? Au-delà de leurs disparités et de leur immaturité, ces "politiques" se rejoignent dans la tentative de transgression des logiques sectorielles et de combinaison du développement économique avec le maintien de la cohésion du tissu social, ce qui peut être un embryon de "sens".

Des outils institutionnels nouveaux, notamment européens avec les programmes LEADER, peuvent appuyer ce type de démarche. Même si les exemples analysés montrent un "outil" encore balbutiant, technocratique et soumis à la pesanteur des gestions notabilaires locales, ces programmes représentent des embryons de "politiques rurales" (Chalmain, 
1993). Globalement, les opportunités offertes à l'agriculture par ces nouveaux modes d'insertion se traduisent en termes de financements, d'appui technique à l'élaboration des projets, d'appui à la formation ; mais aussi, de manière probablement plus déterminante et durable, en terme d'ouverture sur de nouveaux partenariats, de création de synergies locales, sources de développement économique et d'emploi diffus. Peut-on voir là des embryons de "districts ruraux", à l'image des "districts industriels marschalliens" ? (Benko, Lipietz, 1992).

\section{Conclusion}

$\mathbf{U}$ $n$ référentiel alternatif est donc en train de se structurerdans l'agriculture des zones défavorisées, sur la base d'une insertion renforcée dans le marché, et d'une ouverture sur les opportunités fournies par la sphère territoriale ou les réseaux.

Va-t-il se renforcer, se diffuser à une échelle significative? Et si oui, est-il de nature à limiter le déclin agricole de ces zones, ou bien est-il lui-aussi une "soupape sociale" permettant de gèrer leur déclin plus en douceur?

Son émergence spontanée démontre, au-delà des pesanteurs sectorielles, une certaine vitalité de l'agriculture des zones défavorisées. Il élargit le champ des possibles économiques et doit être, à ce titre, soutenu par des politiques rurales adaptées, dont l'Europe propose une esquisse à travers les programmes LEADER.

Cela étant, l'accès à ce référentiel est limité, notamment de par les compétences qu'il suppose. Par ailleurs, ce référentiel reste fragile : d'une part, parce que son organisation, sur une base autonome, est encore embryonnaire; d'autre part, parce qu'il est largement fondé sur les aspirations de la société à l'égard de l'agriculture et de la ruralité (Hervieu, 1993). Des aspirations qui sont encore insuffisamment formulées (Delors, Lacombe, 1993), dont on peut se demander si elles sont "centrales ou périphériques" (Allaire, 1989), et qui, pour l'instant, ont peu de transcriptions institutionnelles claires.

Q i le maintien d'une agriculture diffuse en zone défavori$\checkmark$ sées est reconnu comme nécessaire, ni la forme actuelle de la PAC, ni l'émergence de ce référentiel alternatif ne permettront de faire l'économie d'un double débat :

- l'un portant sur les conditions de consolidation du référentiel alternatif, (quelles politiques rurales ?);

- l'autre portant sur la gestion de la production agricole, et de son rôle dans l'aménagement du territoire.

Betty WAMPFLER

CIRAD-ENSAM, Montpellier.

\section{RÉFÉRENCES BIBLIOGRAPHIQUES}

Allaire G. Le développement rural et les politiques agricoles de transition. Quel paradigme alternatif au productivisme ? INRA-ESR Toulouse, 1989, $33 \mathrm{p}$.

Benko G, Lipietz A. Les régions qui gagnent. Districts et réseaux : les nouveaux paradigmes de la géographie économique. PuF Economie en liberté, 1992, Paris, 419 p.

Bergmann D, Baudin P. Politiques d'avenir pour l'Europe agricole. INRA-Economica, 1989, Paris, $170 \mathrm{p}$.

Bodiguel M. Le rural en question. L'Harmattan, 1986, Paris, $183 \mathrm{p}$.

Bonny S. Vers un autre modèle de développement agricole? Economie rurale, 1981, n 146, p. 20.

Boussard JM. Introduction à l'économie rurale. Cujas, $1992,110 \mathrm{p}$.

Chalmain P. Politiques rurales. In "Agriculture, semer l'avenir", PROJET, 1993, n' 234, p. 7

Coulomb $\mathrm{P}$, Nallet $\mathrm{H}$. Le syndicalisme agricole et la création du paysan modèle. INRA-CORDES, $1980,88 \mathrm{p}$.

De Bandt J. La filière comme méso-système. In Traité d'Economie Industrielle. Economica, 1988, Paris.

Delors B, Lacombe P. Les agriculteurs dans la société. In "Naissance de nouvelles campagnes" dir. Kayser B. DATAR, Editions de l'Aube, 1993, p. 65.

Eizner N. Les paradoxes de l'agriculture française. Essai d'analyse à partir des Etats Généraux du développement agricole. L'Harmattan, 1985, Paris, 159 p.

Guyomard H, Mahe L.P. La réforme de la PAC, une révolution ou un grand pas dans la bonne direction? Session SFER, 1991.

Hervieu B. Les champs du futur. Ed. François Bourin, 1993, $172 \mathrm{p}$.

INRA Col. Réforme de la PAC. Simulation et analyses. INRA Recherches en économie et sociologie rurales, 1992, $n^{\circ} 6$.

Loyat J. La réforme de la PAC: une évaluation par le modèle ECAM. Session SFER, 1991.

Muller P, Faure A, Gerbaux F. Les entrepreneurs ruraux. Agriculteurs, artisans, commerçants, élus locaux. L'Harmattan, 1989, 188p.

Muller P. Les politiques publiques. PuF-Que sais-je ? 1990, $\mathrm{n}^{\circ} 2534,127 \mathrm{p}$.

Pecqueur B. De l'espace fonctionnel à l'espace territoire. Essai sur le développement local. Thèse de doctorat sciences économiques, 1987, Grenoble II, 474 p.

Pour. Agriculture, ruralité, société. Les termes d'un débat. L'Harmattan, revue Pour, 1991, n 130-131.

PROJET. Agriculture, semer l'avenir. Revue PROJET, 1993, $\mathrm{n}^{\circ} 234$.

Touzard JM. Régulation sectorielle, dynamique régionale et transformation d'un système productif localisé : l'exemple de la viticulture languedocienne. In Institution et conventions dans l'agriculture, ed. Allaire G. et Boyer R., INRA Economica, 1993.

Valceschini E. Exploitation, filière et mésosystème. In Modélisation systémique et système agraire, décision et organisation, sous la direction de J. Brossier, B. Vissac, JL. Lemoigne, INRA SAD, 1992, $13 \mathrm{p}$. 